\title{
Chemoradiotherapy in combination with radical surgery is associated with better outcome in cervical cancer patients
}

\author{
Dan Zheng ${ }^{1,2, *}$, Hua-Ping Mou ${ }^{3, *}$, Peng Diao ${ }^{4, *}$, Xiao-Ming Li ${ }^{5}$, Chuan-Li Zhang ${ }^{3}$, Jing \\ Jiang $^{3}$, Jia-Lian Chen ${ }^{3}$, Li-Shuai Wang ${ }^{6}$, Qiu Wang ${ }^{6}$, Guang-Yuan Zhou ${ }^{6}$, Jie Chen ${ }^{6}$, \\ Chuan Lin ${ }^{6}$ and Zhi-Ping Yuan ${ }^{5,6}$ \\ ${ }^{1}$ Department of Head and Neck and Mammary Gland Oncology, and Medical Oncology, Cancer Center, West China Hospital, \\ Sichuan University, Chengdu, Sichuan, 610041, P.R. China \\ ${ }^{2}$ State Key Laboratory of Biotherapy/Collaborative Innovation Center of Biotherapy, West China Hospital, Sichuan University, \\ The Cancer Center, Chengdu, Sichuan, 610041, P.R. China \\ ${ }^{3}$ Department of Gynecology, The Second People's Hospital of Sichuan Province, Yibin City, Yibin, Sichuan, 644000, P.R. China \\ ${ }^{4}$ Sichuan Cancer Hospital and Institute, Sichuan Cancer Center, School of Medicine, 3 University of Electronic Science and \\ Technology of China, Chengdu, Sichuan, 610041, P.R. China \\ ${ }^{5}$ Department of Hematology, The First Affiliated Hospital of Southwest Medical University, Luzhou, Sichuan, 646000, P.R. China \\ ${ }^{6}$ Department of Oncology, The Second People's Hospital of Sichuan Province, Yibin City, Yibin, Sichuan, 644000, P.R. China \\ *These authors contributed equally to this work
}

Correspondence to: Zhi-Ping Yuan, email: yuanzhipingsc@hotmail.com

Keywords: cervical cancer; radical surgery; concurrent chemoradiotherapy (CCRT); clinical outcome

Received: August 31, $2017 \quad$ Accepted: November 16, $2017 \quad$ Published: December 08, 2017

Copyright: Zheng et al. This is an open-access article distributed under the terms of the Creative Commons Attribution License 3.0 (CC BY 3.0), which permits unrestricted use, distribution, and reproduction in any medium, provided the original author and source are credited.

\section{ABSTRACT}

Objectives: To retrospectively assess the influence of radical surgery following concurrent chemoradiotherapy (CCRT) on outcomes in cervical cancer (CC) patients.

Methods: Patients diagnosed with cervical squamous cell carcinoma or adenocarcinoma (FIGO stages IB2 to IIB) at the Yinbin Second People's Hospital between September 2008 and September 2013, were included in this study. Patients were classified into 2 groups based on the treatment received: surgery group (CCRT plus radical surgery) and non-surgery groups (CCRT only). In addition to clinical information, inter-group differences with respect to local control rate (LCR), local recurrence rate (LRR), metastasis rate, overall survival (OS), progress free survival(PFS) and complications were assessed.

Results: A total of $\mathbf{3 1 4}$ patients were included in the analysis. Parametrial invasion, pelvic lymph node metastasis, tumor diameter $>4 \mathrm{~cm}$ and presence of residual disease were risk factors for recurrence in the non-surgery group. In patients with risk factors, radical surgery significantly improved their clinical outcome. The 3-year/5-year LCR in the surgery and non-surgery groups was $\mathbf{8 8 . 3} \% / 87.4 \%$ and $82.3 \% / 77.5 \%$, respectively $(P=0.04)$. The 3 -year $/ 5$-year OS rate in the two groups was $87.1 \% / 81.7 \%$ and $72.8 \% / 67.3 \%$, respectively $(P=0.001)$. The 3 -year $/ 5$-year LRR in the two groups were $11.7 \% / 12.6 \%$ and $17.7 \% / 22.5 \%$, respectively $(P=0.04)$. The metastasis rates in the two groups were $19.9 \%$ and $24.8 \%$, respectively $(P=0.09)$.

Conclusions: Surgery following CCRT could improve overall survival and progressfree survival. Radical surgery following CCRT appears to confer significant benefits including an increase in LCRs and decrease in LRR in CC patients with risk factors.

\section{INTRODUCTION}

Cervical cancer (CC) is the fourth most common cancer worldwide for females, and the seventh most common cancer overall [1]. Many CC patients do not have resources available for undergoing surgery at the time of diagnosis. Tumor bulk, slow shrinkage after radiotherapy or residual disease, parametrial invasion, 
lymph node metastases are known risk factors for CC recurrence [2-5]. Residual disease post chemoradiotherapy refers to both residual disease as well as development of fibrosis. A fraction of patients achieve a complete response (CR) during the follow up period. Treatment modalities for residual disease vary between centers and are controversial $[6,7]$. In some countries, post-radiotherapy radical hysterectomy is a common practice for stage Ib-II CC while another study reported no therapeutic impact in patients achieving CR following CCRT $[8,9]$. The maximum toxicity to normal tissues is exceeded for those patients receiving maximum tolerated doses during the initial chemoradiotherapy phase and interstitial implant branchy therapy can be hampered as a result. Surgery following radiotherapy has been shown to confer a survival benefit and lower local recurrence rate in $\mathrm{CC}$ patients $[8,10]$. The first phase of treatment alters the anatomy of the pelvis tissue around the irradiation field thereby limiting treatment options for any subsequent recurrence. Thus, it is important to follow a comprehensive treatment strategy for reducing recurrence rates in patients at high-risk for recurrence. The present study aims to compare the outcomes between selected patients treated with early radical surgery after CCRT and those treated with CCRT alone.

\section{RESULTS}

\section{Baseline variables}

Data pertaining to 314 eligible patients were retrospectively analyzed. No statistically significant baseline inter-group differences were observed with respect to age (median, 51 vs. 55); Eastern Cooperative Oncology Group (ECOG) scores ; pathological types ; FIGO stages; radiotherapy regimen ; tumor diameters ; pelvic lymph node status ; parametrial infiltration ; and chemotherapy cycles (Table 1). Taken together, only significant difference was observed between the two groups in parametrial infiltration,

\section{Response and recurrence rates}

Tumor response rate after CCRT in the surgery vs non-surgery groups are shown in Supplemental Digital Content Supplementary Table 3. 3-year LCR were $88.3 \%$ and $82.3 \%$, respectively; 5-year LCR were $87.4 \%$ and $77.5 \%(P=0.04)$, respectively. At the endpoint of follow-up, the 3-year local recurrence rate (LRR) and total LRR in the surgery group were $11.7 \%$ and $12.6 \%$, respectively, and in the non-surgery group were $17.7 \%$ and $22.5 \%$, respectively $(P=0.04)$ (Figure 1$)$. Furthermore, the LRR in patients with parametrial invasion $(P=$ $0.04)$, positive pelvic lymph nodes $(P=0.003)$, residual disease $(P=0.03)$ and tumor size $>4 \mathrm{~cm}(P=0.03)$ were significantly higher than without these factors in the non-surgery group (Figure 2 and Supplementary Digital Content Supplementary Table 1). However, there were no statistically significant differences in LRR between patients with or without risk factors, including parametrial invasion $(P=0.25)$, pelvic lymph node metastases $(P=$ $0.67)$, residual diseases $(P=0.71)$ and tumor diameter $>4 \mathrm{~cm}(P=0.29)$ in the surgery group (Figure 2$)$. These results revealed that radical operation appears to significantly reduce the recurrence rate in patients with these risk factors. In the non-surgery group, 18 patients experienced LR including 11 parametrical recurrences and 7 primary recurrences. Whereas in the surgery group, 12 patients suffered LR including 6 parametrical recurrences and 6 primary recurrences. However, there was no significant difference in distant metastases rate (DMR) between the two groups. 3-year and 5-year DMR were $23.8 \%$ and $24.8 \%$ in the non-surgery group while $16.3 \%$ and $19.9 \%$ in the surgery group, respectively $(P=$ $0.09)$. In the surgery group, 14 patients $(8.59 \%)$ suffered lung metastases, 10 patients $(6.13 \%)$ had supraclavicular metastases, 5 patients $(3.07 \%)$ had para aortic lymph node metastases and 7 patients (4.29\%) had both local/ regional and distant metastases. In the non-surgery group, 16 patients $(10.60 \%)$ suffered lung metastases, 8 patients $(5.30 \%)$ had supraclavicular metastases, 8 patients $(5.30 \%)$ had para aortic lymph node metastases and 8 patients $(5.30 \%)$ had both local/regional and distant metastases.

\section{Outcomes of post operation histological examination}

Histological examination after surgery demonstrated that 56 patients $(34.4 \%)$ had parametrical invasion, 65 patients $(39.9 \%)$ had residual disease, and 12 patients $(7.4 \%)$ had positive margin. Whereas 13 patients whose enhanced CT or MRI show that there was residual disease but was not confirmed by histology. Furthermore, 41 patients $(25.2 \%)$ showed pelvic lymph node metastases, 14 patients whose image records demonstrated positive pelvic lymph node metastases turn out to be negative in histological examination. In addition, 3.7\% patients had lymphatic vessel invasion (LVSI) as determined by histological analysis.

\section{Overall survival (OS) and progress free survival (PFS)}

The 3-year/5-year OS rate in the surgery group was $87.1 \%$ and $81.7 \%$, respectively; while the corresponding Figures in the non-surgery group were $72.8 \%$ and $67.3 \%$, respectively $(P=0.001$, Figure 3$)$. For patients with parametrial invasion, the 3-year/5-year OS rate in the surgery group was $89.6 \%$ and $84.6 \%$, respectively; while the corresponding Figures in the non-surgery group, were $61.9 \%$ and $56.4 \%$, respectively $(P<0.0001)$ (Figure 


\begin{tabular}{|c|c|c|c|}
\hline Characteristics & $\begin{array}{l}\text { Surgery group } \\
\quad(N=163)\end{array}$ & $\begin{array}{c}\text { Non-surgery } \\
\text { group }(N=151)\end{array}$ & $P$ value \\
\hline $\begin{array}{l}\text { Ages } \\
\quad \text { Median (range) }\end{array}$ & $51(26-73)$ & $55(28-79)$ & $0.48^{*}$ \\
\hline ECOG scores & & & $0.89^{\#}$ \\
\hline$\leq 2(N, \%)$ & $157,96.3 \%$ & $145,96.0 \%$ & \\
\hline$>2(N, \%)$ & $6,3.7 \%$ & $6,4.0 \%$ & \\
\hline Pathological types & & & $0.45^{\#}$ \\
\hline $\operatorname{SCC}(N, \%)$ & $136,83.4 \%$ & $121,80.1 \%$ & \\
\hline $\operatorname{AC}(N, \%)$ & $27,16.6 \%$ & $30,19.9 \%$ & \\
\hline FIGO stages & & & $0.20^{\#}$ \\
\hline IB2 $(N, \%)$ & $35,21.6 \%$ & $28,18.5 \%$ & \\
\hline $\operatorname{IIA} 1(N, \%)$ & $27,16.6 \%$ & $39,25.8 \%$ & \\
\hline IIA2 $(N, \%)$ & $44,27.0 \%$ & $34,22.5 \%$ & \\
\hline IIB1 $(N, \%)$ & $41,25.2 \%$ & $30,19.9 \%$ & \\
\hline IIB2 $(N, \%)$ & $16,9.8 \%$ & $20,13.2 \%$ & \\
\hline Radiotherapy schemes & & & $0.08^{\#}$ \\
\hline $\operatorname{IMRT}(N, \%)$ & $100,61.3 \%$ & $78,51.7 \%$ & \\
\hline 3D-CRT $(N, \%)$ & $63,38.7 \%$ & $73,48.3 \%$ & \\
\hline Tumor diameters & & & $0.14^{\#}$ \\
\hline$\leq 4 \mathrm{~cm}(N, \%)$ & $103,68.2 \%$ & $103,68.2 \%$ & \\
\hline$>4 \mathrm{~cm}(N, \%)$ & $60,36.8 \%$ & $48,31.8 \%$ & \\
\hline Pelvic lymph node status & & & $0.16^{\#}$ \\
\hline Positive $(N, \%)$ & $60,36.8 \%$ & $41,25.2 \%$ & \\
\hline Negative $(N, \%)$ & $103,63.2 \%$ & $110,72.8 \%$ & \\
\hline Parametrial invasion & & & $0.03^{\#}$ \\
\hline Yes $(N, \%)$ & $56,34.4 \%$ & $70,46.4 \%$ & \\
\hline $\operatorname{No}(N, \%)$ & $107,65.6 \%$ & $81,53.6 \%$ & \\
\hline Chemotherapy & & & $0.19^{\#}$ \\
\hline 4 cycles $(N, \%)$ & $13,8.0 \%$ & $19,12.6 \%$ & \\
\hline 5 cycles $(N, \%)$ & $31,19.0 \%$ & $31,20.5 \%$ & \\
\hline 6 cycles $(N, \%)$ & $119,73.0 \%$ & $62,66.9 \%$ & \\
\hline
\end{tabular}

"Mann-Whitney test; "Fisher's exact test; IMRT, intensity-modulated radiotherapy; 3D-CRT, three-dimensional conformal radiotherapy; ECOG, Eastern Cooperative Oncology Group.

4A). For patients with positive pelvic lymph nodes the 3 -year $/ 5$-year OSR was $85.2 \%$ and $73.1 \%$ in the surgery group, respectively; while it was $64.7 \%$ and $60.1 \%$ in the non-surgery group, respectively $(P=0.09)$ (Figure 4B). In patients with residual disease, the 3 -year/5-year OSR in the surgery group was $79.4 \%$ and $74.3 \%$, respectively; while it was $63.3 \%$ and $61.0 \%(P=0.02)$ in the non-surgery group (Figure 4C). Moreover, in patients with tumor diameter $>4 \mathrm{~cm}$ the 3 -year/5-year OSR in the surgery group was $84.1 \%$ and $76.0 \%$, respectively; whereas in the non-surgery groups the corresponding Figures were $80.4 \%$ and $74.3 \%$, respectively $(P=0.56)$ (Figure 4D). These results indicate that patients with risk factors including parametrical invasion and residual disease may benefit from radical operation following chemoradiotherapy as the OS were significantly increased in the surgery group compared to the non-surgery group. However, survival benefits brought about by surgery were considerable but did not reach statistical significance in patients with pelvic lymph node metastases and bulk tumor size. The 3-year/5year PFSR in the surgery group was $77.3 \%$ and $73.3 \%$, respectively; while the corresponding Figures in the nonsurgery group were $67.2 \%$ and $62.4 \%$, respectively $(P=$ $0.01)$. Moreover, PFS was significantly higher in patients 
with these four recurrence risk factors in the surgery group compared to the non-surgery group (Figure 5).

\section{Univariate and multivariate analysis}

Factors including stage $(\mathrm{RR}=1.41, P=0.008)$, chemotherapy cycle $(\mathrm{RR}=0.48, P=0.001)$, parametrical invasion $(\mathrm{RR}=2.08, P=0.02)$ and tumor diameter $>4$ $\mathrm{cm}(\mathrm{RR}=2.03, P=0.03)$ were correlated with recurrence in univariate analysis by ANOVA (analysis of variance). Factors including parametrical invasion $(\mathrm{RR}=2.03, P$ $=0.04)$, tumor diameter $>4 \mathrm{~cm}(\mathrm{RR}=2.35, P=0.01)$, stage $(\mathrm{RR}=1.33, P=0.03)$ and chemotherapy cycle $(\mathrm{RR}$ $=0.47, P=0.001)$ showed significant correlations with local recurrence in multivariate analysis by MANOVA (multivariate analysis of variance).

\section{Toxicity of CCRT and surgical complications}

Hematological toxicity of grades 1, 2, 3 and 4 were documented in 289 patients, including 92 cases (29.3\%), 137 cases (43.6\%), 40 cases (12.7\%) and 6 cases $(1.9 \%)$, respectively. Febrile neutropenia was reported in 2 patients. Grade 1, 2, 3 radiation-induced enteritis and proctitis were reported in 79 cases $(25.2 \%), 13$ cases $(4.1 \%)$, and 8 cases $(2.6 \%)$, respectively. Furthermore, $8(2.6 \%)$ and $7(2.2 \%)$ patients experienced grade 1 and grade 2 radiodermatitis, respectively; and grade 1 irradiation cystitis was reported in $10(3.18 \%)$ patients. We did not observe grade 5 toxicity in our study, and none of the patients died of CCTR toxicity. Nineteen $(11.7 \%)$ patients who suffered from urinary retention recovered from this side effect within 3 weeks after radical operation with bladder functional exercises.
Collectively, these results suggest that recurrence rates may be reduced and OS and PFS increased by performing radical operation following CCRT in patients who have recurrence risk factors mentioned in this study. Patients with these risk factors had significantly lower recurrence in the surgery group as compared to that in the non-surgery group. Moreover, differences in recurrence rates can be reduced by radical operation. Among high risk patients, recurrence rates were similar in the surgery group, but they were significantly different in the nonsurgery group.

\section{DISCUSSION}

Although CCRT, the current standard of care treatment regimen for advanced CC, significantly increase OS and PFS compared to the radiotherapy alone [11], there remain risk factors leading to poor response to CCRT and increase in recurrence. There was no significant difference in the effect of CCRT between the two groups (Supplementary Table 3). Outcomes of CCRT in the two groups in the present study were comparable to other studies with similar dosing regimens of radiotherapy and chemotherapy [12-14]. All patients in our study had one or more recurrent risk factor mentioned above. The surgery group in our study had significantly lower LRR and the benefit brought about by LC partially translate into an increase in OS. A previous report observed $16.7 \%$ recurrence in patients undergoing surgery following CCRT compared to $31.7 \%$ recurrence of those that did not undergo surgery [15]. However, surgery following CCRT remains controversial because of severe complications. Coleman et al reported $38.2 \%$ severe complications in patients receiving radical

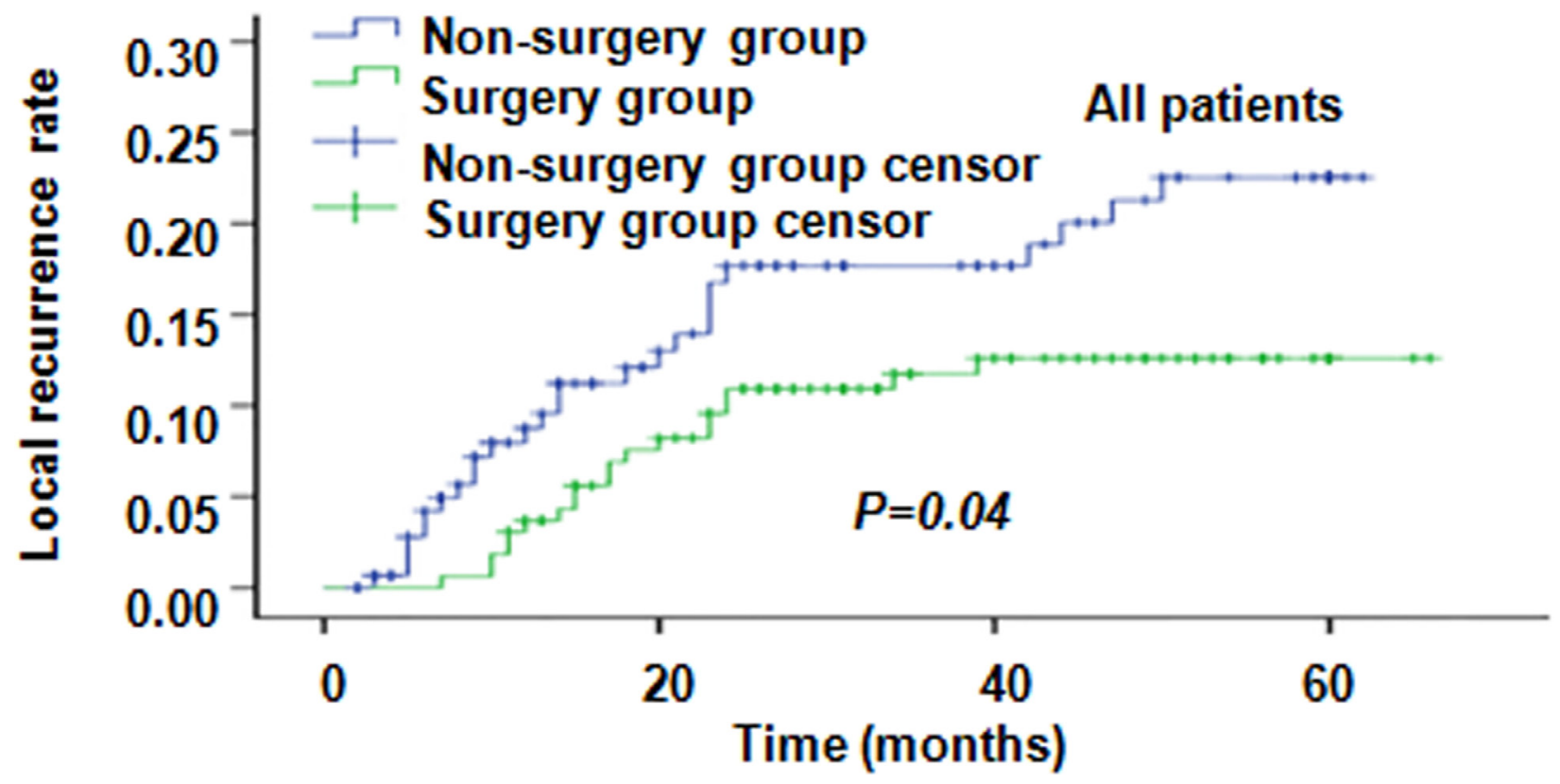

Figure 1: Local recurrence rates (LRR) in all the patients. 
hysterectomy and lymphadenectomy [16]. LR is the main reason for treatment failure that may translate to distant metastases (DM) [17]. The aim of adding surgery is not to reduce the toxicity induced by CCRT through decreasing the doses used during CCRT, but to increase the LC of patients with recurrence risk factors. In the present study, we observe a significantly higher LC that translated into a partial survival gain. However, with regard to surgery after chemoradiotherapy, interval time between CCRT and surgery and surgery type are not clear. First, a fraction of DM occurrences may not be located using modern radiology technology while detecting DM using biopsy also has limitations. Vandeperre A et al. reported 5\% in $\mathrm{PET} / \mathrm{CT}, 13 \%$ in $\mathrm{PET}$ and $6 \%$ in $\mathrm{CT}$ are false negative in para aortic lymph node metastases [18]. Therefore, we cannot absolutely exclude micro DM in the surgery group. Moreover, PD after CCRT should not be given surgery, however one patient with $\mathrm{PD}$ received surgery because a new pelvic lymph node metastases had been found in MRI. Second, the interval time between CCRT and surgery is controversial. Surgery after CCRT is implemented 4-8 weeks in studies including rectal cancer and CC $[17,19]$. This is because fibrosis is a late adverse event occurring 3 months after CCRT that leads to
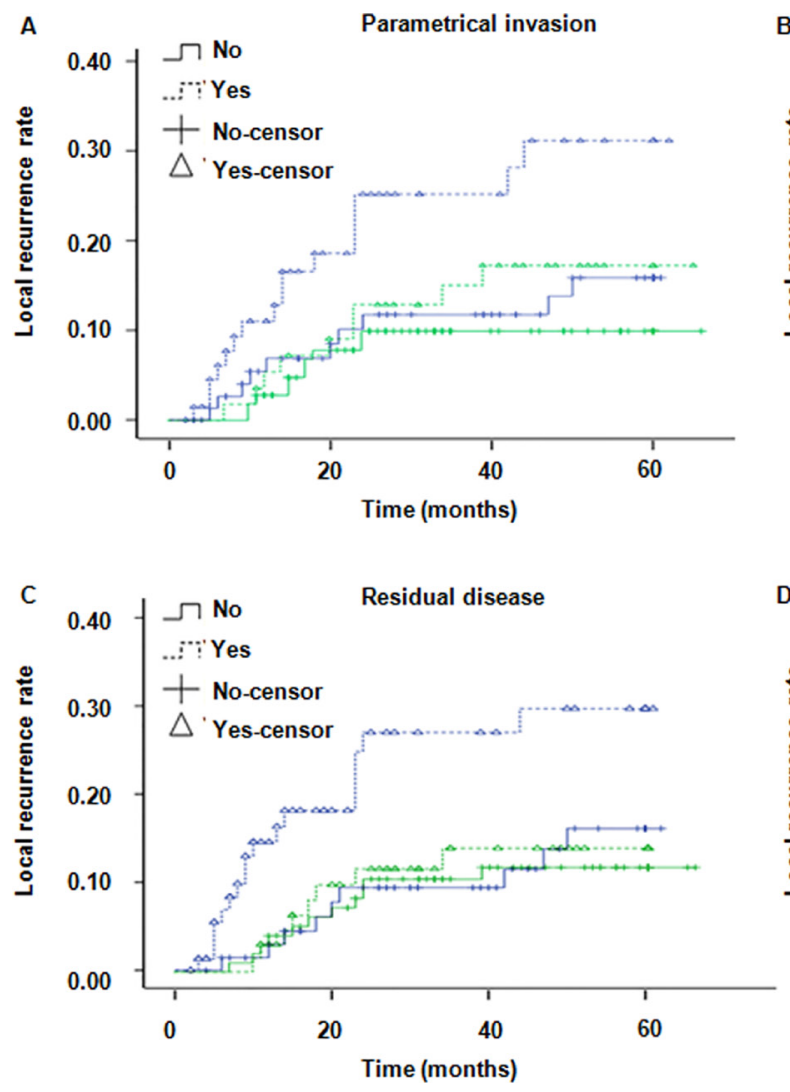

Figure 2: LRR in patients with or without risk factors. Green line and blue line represent the surgery group and the non-surgery group, respectively. (A) LRR in patients with or without parametrial invasion $(P$ value for surgery and non-surgery groups were 0.25 and 0.04 , respectively); (B) LRR in patients with or without pelvic lymph node metastases ( $P$ value for surgery and non-surgery groups were 0.67 and 0.003 , respectively); (C) LRR in patients with or without residual disease ( $P$ value for surgery and non-surgery groups were 0.71 and 0.03 , respectively); (D) LRR in patients with or without tumor diameter $>4 \mathrm{~cm}$ ( $P$ value for surgery and non-surgery groups were 0.29 and 0.03 , respectively.).
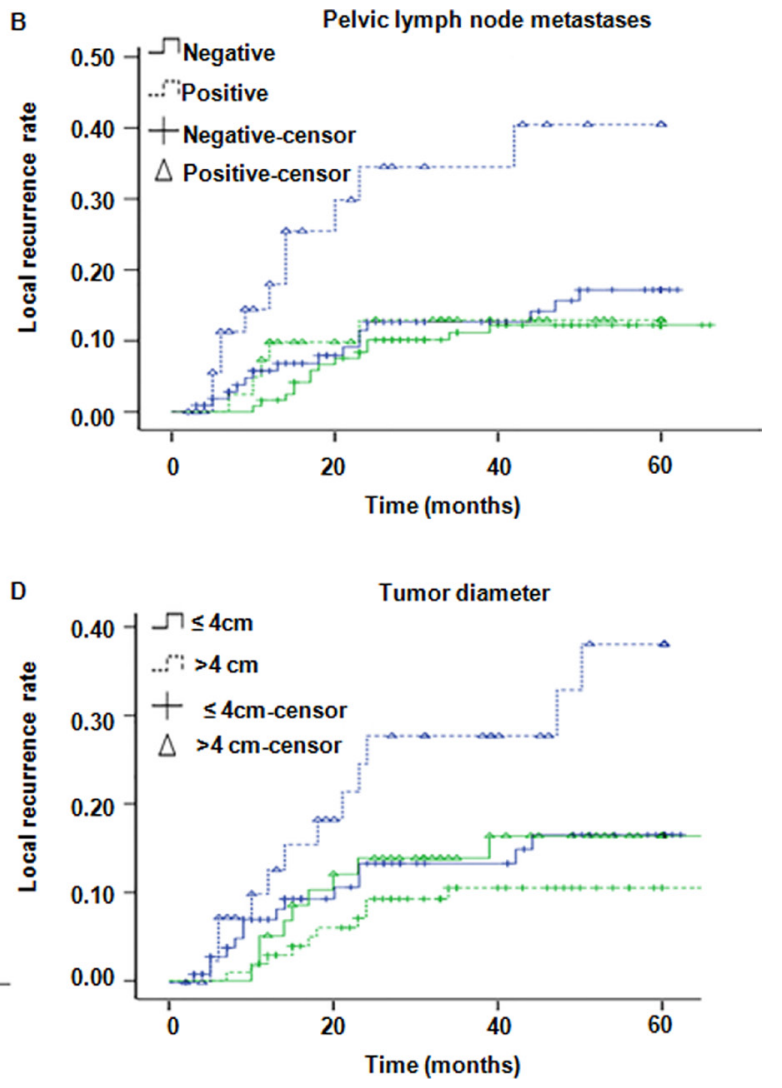

difficulty in subsequent surgery [20-22]. A small number of patients received surgery within 3-4 weeks due to the patients' requirement in our study. We found that surgery within 4 weeks following CCRT does not increase the surgery complications because edema induced by radiation mild by this time. Third, laparoscopic hysterectomy and arotomy were included in the present study. There were es of surgery except that complications, length of stay such as extrafascial hysterectomy, radical hysterectomy and extended hysterectomy. Sun L et al. reported that the most appropriate surgical approach is extrafascial hysterectomy, which had a significantly lower incidence of postoperative complication [15].

The use of surgery after CCRT is a controversial topic. To our knowledge, studies examining the effect of CCRT combined with surgery are quite limited. Houvenaeghel et al. reported a 10 -year OS rate of $57.7 \%$ in these patients [23]. Another study demonstrated that CCRT combined with surgery can significantly reduce recurrence and extend survival with no severe surgical complications [24]. Our results indicate that the difference 


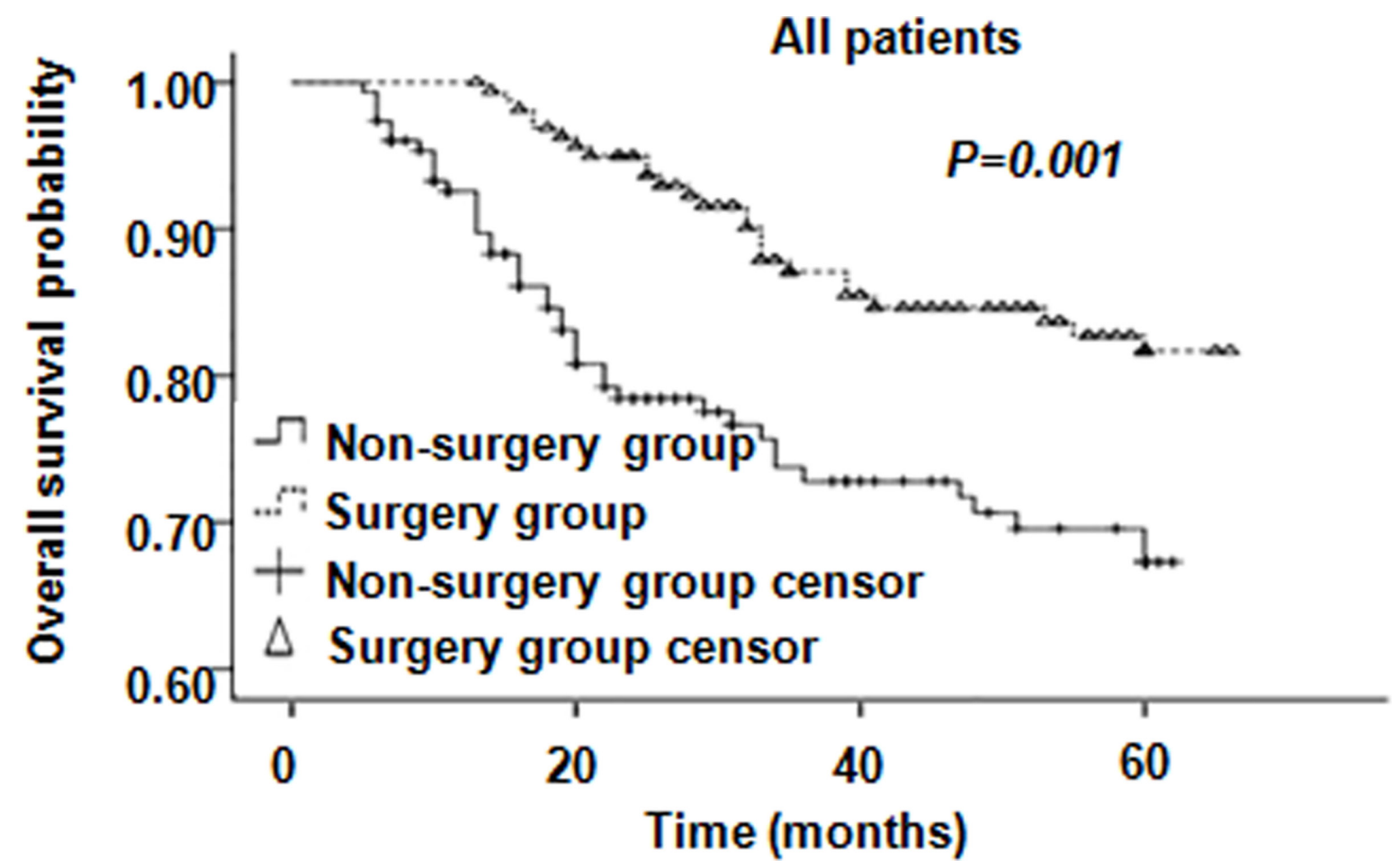

Figure 3: Overall survival of all patients. There is no difference in OS between surgery and non-surgery groups.
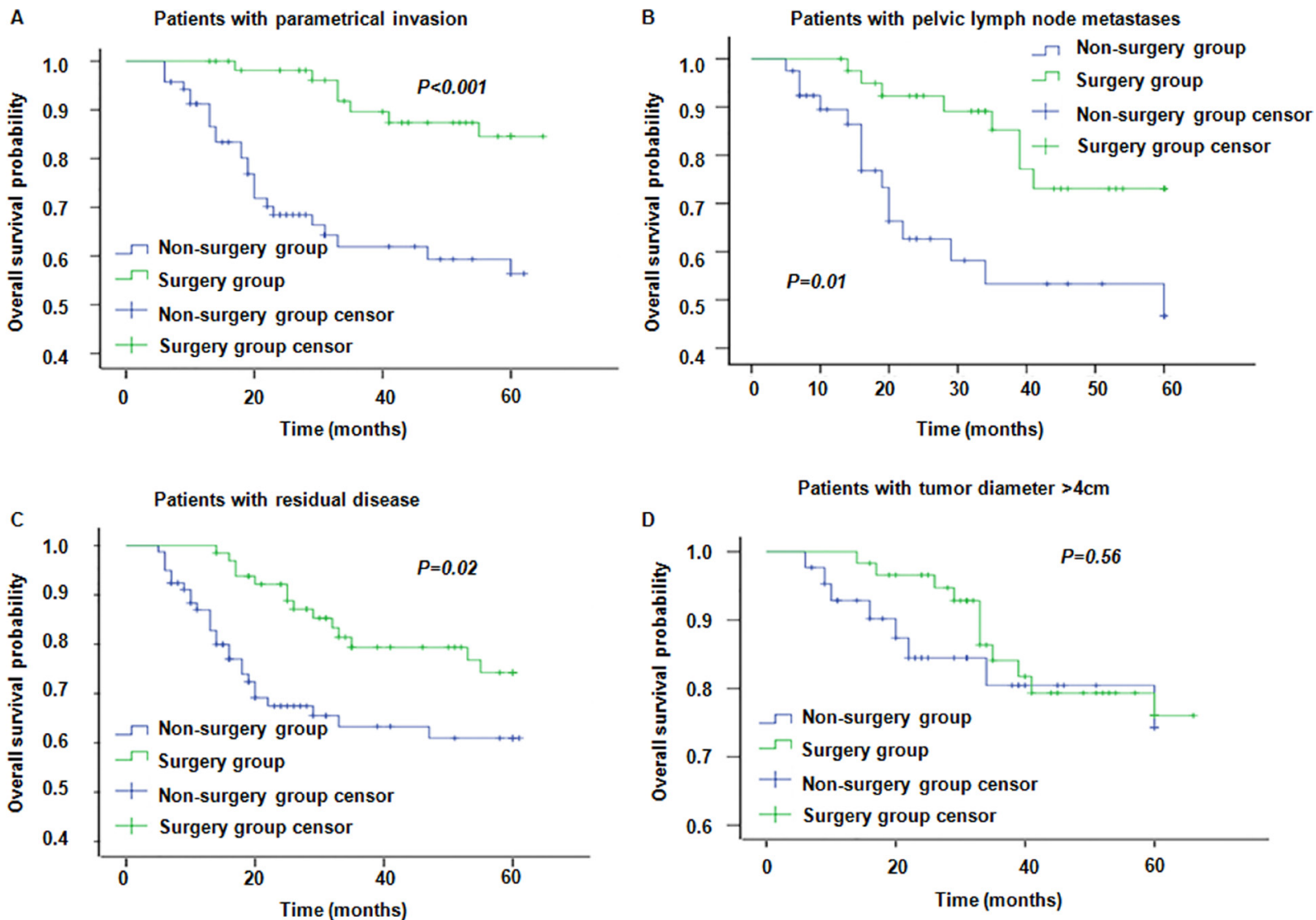

Figure 4: Overall survival in patients with different risk factors in surgery group and non-surgery group. (A-D) represent OS for patients with parametrical invasion, pelvic lymph node metastases, residual disease and tumor diameter $>4 \mathrm{~cm}$, respectively. 
in recurrence between patients with and without risk factors can be significantly decreased by radical surgery (see Figure 2). Parametrical invasion and tumor diameter $>4 \mathrm{~cm}$ are important risk factors associated with prognosis (Supplemental Digital Content Supplementary Table 2). The recurrence rate was lower in the surgery group, while the risk factors contributed to LR in the non-surgery group (Figure 2). To our knowledge, this is the first study to show surgery significantly improves OS and PFS. The aim of surgery as part of a multidisciplinary treatment strategy is to achieve LC by resecting the primary lesion, and clearing or decreasing the tumor load, while chemotherapy serves to treat the circulating tumor cells (CTCs) and DM [25-30]. We find it is likely that surgery decreases local/ regional recurrence rate and lymph node metastases which may contribute to the increase in OS and PFS rather than post-operation surgery increasing survival time directly as the DM rate is similar between the two groups. Tumor bulk and residual disease after radiotherapy are associated with lymph node and DM, and surgery can confer survival benefit in these patients $[31,32]$.

In summary, our results suggest that early and proactive radical surgery can help decrease incidence of LR and prolong OS in patients with risk factors for
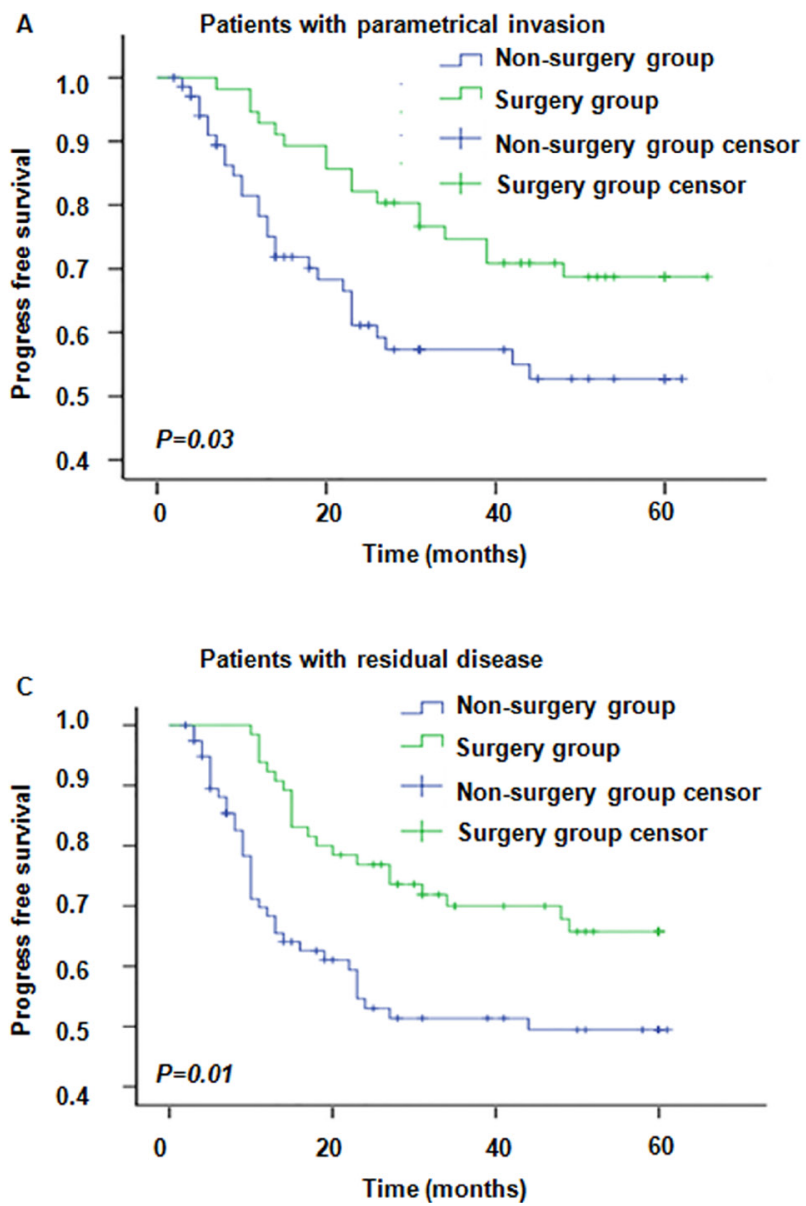

recurrence. Future studies with larger sample sizes are needed to confirm the clinical recommendation of radical surgery following CCRT.

\section{MATERIALS AND METHODS}

\section{Patients}

Patients diagnosed with cervical squamous cell carcinoma (SCC) and adenocarcinoma (AC) by cytology or histology, and classified as FIGO stage IB2-IIB with high recurrence risks assessed by gynecological examination and radiography between September 2008 and September 2013 were enrolled. Tumor diameter was measured on enhanced CT or MRI on three orthogonal plans of T2-weighted image and maximum axis was chosen. Parametrical invasion was defined as a disrupted stromal ring surrounding the uterus or parametrium irregular signal intensity in T2-weighted or diffusion-weighted image [33, 34]. Pelvic lymph node metastases were defined as high signal intensity or the shortest axis $>5 \mathrm{~mm}$ in abdominal pelvic CT or MRI [35]. In addition, patients who had residual disease on CT or MRI after CCRT underwent a biopsy and those with positive biopsy were scored as residual disease
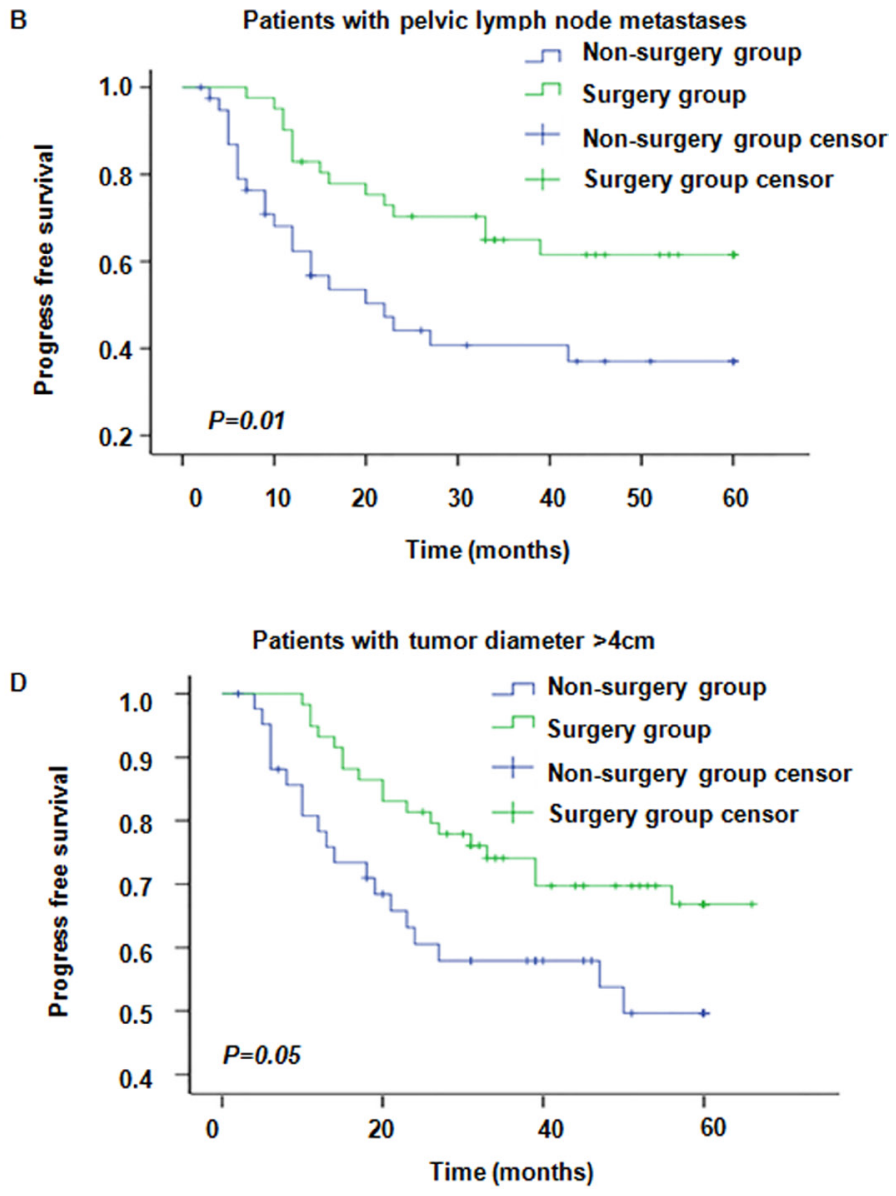

Figure 5: Progress free survival in patients with different risk factors in surgery and non-surgery groups. (A-D) represent PFS for patients with parametrical invasion, pelvic lymph node metastases, residual disease and tumor diameter $>4 \mathrm{~cm}$, respectively. 
[36]. Residual disease was defined as definite nodes or irregular signal intensity in the primary tumor site on MRI or high signal intensity on enhanced CT scan. All patients received CCRT in Yinbin Second People's Hospital and a proportion of patients did not undergo radical operation due to economic and other complications.

Patients were categorized into surgery or non-surgery groups based on the treatment received, i.e., radical surgery following CCRT and CCRT alone, respectively. Patients underwent gynecological examination, routine blood examination, blood biochemistry, serum SCC-Ag, CEA, CA-125, liquid-based cytology or biopsy, plain chest CT, inferior abdomen and pelvic enhanced MRI at admission.

Patients with any of the following features were excluded from the study: pathological type other than $\mathrm{SCC}$ or AC, severe anemia (HGB $<60 \mathrm{~g} / \mathrm{L}$ ) pretreatment or intra-treatment, history of inferior abdominal or pelvic exenteration or irradiation, cervical stump cancer, incomplete treatment, or incomplete records.

The study was approved by the ethics committee at the Yibin Second People's Hospital. Informed consent was obtained from all patients enrolled.

\section{Concurrent chemoradiotherapy (CCRT)}

A 6MV x-ray beam delivered by an Elekta Precise medical linear accelerator was used for external beam radiation therapy (EBRT). The radiation treatment plan consisted of 3D-CRT and IMRT. EBRT with a total pelvic dose of 46-50 Gy traditional fraction was combined with a total dose of 25-30 Gy, 5-6 Gy per fraction once a week intracavitary brachytherapy on a different day. Cisplatinum $\left(40 \mathrm{mg} / \mathrm{m}^{2}\right)$ was delivered once a week during the period of radiotherapy for 6 weeks. Patients were evaluated weekly with measurements of blood count, blood biochemistry, liver and renal function and clinical examination during CRT. If the white blood cell count was $\leq 2 \times 10^{9} / \mathrm{L}$ or neutrophil cell count was $1 \times 10^{9} / \mathrm{L}$ or platelet cell count was $\leq 50 \times 10^{9} / \mathrm{L}$, CCRT was stopped and recombinant human granulocyte colony-stimulating factor (rhG-CSF) and interleukin-11 were administered until the indices recovered. Vaginal douching was done daily from the beginning of radiotherapy to 1.5 years after the completion of radiotherapy to promote epidermal healing and avoid vaginal adhesions.

\section{Response evaluation}

The tumor response was evaluated every two chemotherapy cycles according to Response Evaluation Criteria in Solid Tumors (RECIST version 1.1) until progress or recurrence or the last chemotherapy cycle.

\section{Radical surgery}

Patients underwent a chest plant CT scan, abdominal pelvic enhancement $\mathrm{CT}$ or MRI or PET/CT before surgery and those PS 0-1 without severe surgery contraindication and distant metastases were treated by radical hysterectomy and pelvic lymphadenectomy within 8 weeks after CCRT completion. Biopsy was performed on patients who had scored positive for residual disease by imaging. Notable, those patients who had residual disease on image but confirmed false positive by biopsy without other recurrence risk factors were excluded. All resected tissues were sent for pathological examination.

\section{Follow-up}

All patients had follow-up examinations every 3-6 months during the first two years, and every 6-12 months during the following 3-5 years. Physical examination, gynecological examination, routine blood test, blood biochemistry, SCC, CEA, CA-125, cervical liquid-based cytology or biopsy, pelvic enhanced MRI and plain chest CT were included in the follow-up.

\section{End points and statistical analysis}

End points included LRR, OS, PFS and DMR. Criteria for toxicity of treatment were based on CTCAE V4.0. Statistical analyses were performed with SPSS 19.0. $P$ value $<0.05$ was considered to be statistically significant.

\section{ACKNOWLEDGMENTS AND FUNDING}

We thank our colleague for their critical comments and support. This work was supported by Sichuan Yibin government fund (No. 2001SF009).

\section{CONFLICTS OF INTEREST}

None.

\section{REFERENCES}

1. Vaccarella S, Lortet-Tieulent J, Plummer M, Franceschi $\mathrm{S}$, Bray F. Worldwide trends in cervical cancer incidence: impact of screening against changes in disease risk factors. Eur J Cancer. 2013; 49:3262-3273.

2. Kato T, Takashima A, Kasamatsu T, Nakamura K, Mizusawa J, Nakanishi T, Takeshima N, Kamiura S, Onda T, Sumi T, Takano M, Nakai H, Saito T, et al. Clinical tumor diameter and prognosis of patients with FIGO stage IB1 cervical cancer (JCOG0806-A). Gynecol Oncol. 2015; 137:34-39.

3. Liu J, Li J, Chen Y, Guan X, Wu X, Hao C, Sun Y, Wang Y, Wang X. Tumor-stroma ratio is an independent predictor for survival in early cervical carcinoma. Gynecol Oncol. 2014; 132:81-86.

4. Mayr NA, Wang JZ, Lo SS, Zhang D, Grecula JC, Lu L, Montebello JF, Fowler JM, Yuh WT. Translating 
response during therapy into ultimate treatment outcome: a personalized 4-dimensional MRI tumor volumetric regression approach in cervical cancer. Int J Radiat Oncol Biol Phys. 2010; 76:719-727.

5. Tseng JY, Yen MS, Twu NF, Lai CR, Horng HC, Tseng CC, Chao KC, Juang CM. Prognostic nomogram for overall survival in stage IIB-IVA cervical cancer patients treated with concurrent chemoradiotherapy. Am J Obstet Gynecol. 2010; 202:174 e171-177.

6. Boers A, Arts HJ, Klip H, Nijhuis ER, Pras E, Hollema H, Wisman GB, Nijman HW, Mourits MJ, Reyners AK, de Bock GH, Thomas G, van der Zee AG. Radical surgery in patients with residual disease after (chemo)radiation for cervical cancer. Int J Gynecol Cancer. 2014; 24:1276-1285.

7. Tangjitgamol S, Katanyoo K, Laopaiboon M, Lumbiganon P, Manusirivithaya S, Supawattanabodee B. Adjuvant chemotherapy after concurrent chemoradiation for locally advanced cervical cancer. Cochrane Database Syst Rev. 2014; CD010401.

8. Mazeron R, Gilmore J, Dumas I, Champoudry J, Goulart J, Vanneste B, Tailleur A, Morice P, Haie-Meder C. Adaptive 3D image-guided brachytherapy: a strong argument in the debate on systematic radical hysterectomy for locally advanced cervical cancer. Oncologist. 2013; 18:415-422.

9. Castelnau-Marchand P, Chargari C, Bouaita R, Dumas I, Farha G, Kamsu-Kom L, Rivin Del Campo E, Martinetti F, Morice P, Haie-Meder C, Mazeron R. What to expect from immediate salvage hysterectomy following concomitant chemoradiation and image-guided adaptive brachytherapy in locally advanced cervical cancer. Cancer Radiother. 2015; 19:710-717.

10. Classe JM, Rauch P, Rodier JF, Morice P, Stoeckle E, Lasry S, Houvenaeghel G. Surgery after concurrent chemoradiotherapy and brachytherapy for the treatment of advanced cervical cancer: morbidity and outcome: results of a multicenter study of the GCCLCC (Groupe des Chirurgiens de Centre de Lutte Contre le Cancer). Gynecol Oncol. 2006; 102:523-529.

11. Green JA, Kirwan JM, Tierney JF, Symonds P, Fresco L, Collingwood M, Williams CJ. Survival and recurrence after concomitant chemotherapy and radiotherapy for cancer of the uterine cervix: a systematic review and meta-analysis. Lancet. 2001; 358:781-786.

12. Kim TE, Park BJ, Kwack HS, Kwon JY, Kim JH, Yoon SC. Outcomes and prognostic factors of cervical cancer after concurrent chemoradiation. J Obstet Gynaecol Res. 2012; 38:1315-1320.

13. Chen JL, Huang CY, Huang YS, Chen RJ, Wang CW, Chen YH, Cheng JC, Cheng AL, Kuo SH. Differential clinical characteristics, treatment response and prognosis of locally advanced adenocarcinoma/adenosquamous carcinoma and squamous cell carcinoma of cervix treated with definitive radiotherapy. Acta Obstet Gynecol Scand. 2014; 93:661-668.

14. Ma R, Zhang H, Zou L, Qu Y. Outcomes and prognostic factors of distant metastasis in patients with advanced cervical squamous cell carcinoma treated with concurrent chemoradiotherapy. Zhonghua Fu Chan Ke Za Zhi. 2015; 50:125-130.

15. Sun L, Sheng X, Jiang J, Li X, Liu N, Liu Y, Zhang T, Li D, Zhang X, Wei P. Surgical morbidity and oncologic results after concurrent chemoradiation therapy for advanced cervical cancer. Int J Gynaecol Obstet. 2014; 125:111-115.

16. Colombo PE, Bertrand MM, Gutowski M, Mourregot A, Fabbro M, Saint-Aubert B, Quenet F, Gourgou S, Kerr C, Rouanet P. Total laparoscopic radical hysterectomy for locally advanced cervical carcinoma (stages IIB, IIA and bulky stages IB) after concurrent chemoradiation therapy: surgical morbidity and oncological results. Gynecol Oncol. 2009; 114:404-409.

17. Jurado M, Martinez-Monge R, Garcia-Foncillas J, Azinovic I, Aristu J, Lopez-Garcia G, Brugarolas A. Pilot study of concurrent cisplatin, 5-fluorouracil, and external beam radiotherapy prior to radical surgery $+/$ - intraoperative electron beam radiotherapy in locally advanced cervical cancer. Gynecol Oncol. 1999; 74:30-37.

18. Vandeperre A, Van Limbergen E, Leunen K, Moerman P, Amant F, Vergote I. Para-aortic lymph node metastases in locally advanced cervical cancer: Comparison between surgical staging and imaging. Gynecol Oncol. 2015; 138:299-303.

19. Jung KU, Kim HC, Park JO, Park YS, Park HC, Choi DH, Cho YB, Yun SH, Lee WY, Chun HK. Adjuvant chemotherapy after neoadjuvant chemoradiation and curative resection for rectal cancer: is it necessary for all patients? J Surg Oncol. 2015; 111:439-444.

20. Bronova I, Smith B, Aydogan B, Weichselbaum RR, Vemuri K, Erdelyi K, Makriyannis A, Pacher P, Berdyshev EV. Protection from Radiation-Induced Pulmonary Fibrosis by Peripheral Targeting of Cannabinoid Receptor-1. Am J Respir Cell Mol Biol. 2015; 53:555-562.

21. Miles T, Johnson N. Vaginal dilator therapy for women receiving pelvic radiotherapy. Cochrane Database Syst Rev. 2014; CD007291.

22. Cartwright-Alcarese F. Addressing sexual dysfunction following radiation therapy for a gynecologic malignancy. Oncol Nurs Forum. 1995; 22:1227-1232.

23. Houvenaeghel G, Lelievre L, Buttarelli M, Jacquemier J, Carcopino X, Viens P, Gonzague-Casabianca L. Contribution of surgery in patients with bulky residual disease after chemoradiation for advanced cervical carcinoma. Eur J Surg Oncol. 2007; 33:498-503.

24. Endo D, Todo Y, Okamoto K, Minobe S, Kato H, Nishiyama N. Prognostic factors for patients with cervical cancer treated with concurrent chemoradiotherapy: a retrospective analysis in a Japanese cohort. J Gynecol Oncol. 2015; 26:12-18.

25. Liang JA, Chen SW, Hung YC, Yeh LS, Chang WC, Lin WC, Chang YY. Low-dose, prophylactic, extended-field, intensity-modulated radiotherapy plus concurrent weekly 
cisplatin for patients with stage IB2-IIIB cervical cancer, positive pelvic lymph nodes, and negative para-aortic lymph nodes. Int J Gynecol Cancer. 2014; 24:901-907.

26. Benedetti Panici P, Bellati F, Pastore M, Manci N, Musella A, Pauselli S, Angelucci M, Muzii L, Angioli R. An update in neoadjuvant chemotherapy in cervical cancer. Gynecol Oncol. 2007; 107:S20-22.

27. Choi CH, Kim TJ, Lee JW, Kim BG, Lee JH, Bae DS. Phase II study of neoadjuvant chemotherapy with mitomycin-c, vincristine and cisplatin (MVC) in patients with stages IB2IIB cervical carcinoma. Gynecol Oncol. 2007; 104:64-69.

28. Yin M, Zhao F, Lou G, Zhang H, Sun M, Li C, Hou Y, Li $\mathrm{X}$, Meng F, Chen X. The long-term efficacy of neoadjuvant chemotherapy followed by radical hysterectomy compared with radical surgery alone or concurrent chemoradiotherapy on locally advanced-stage cervical cancer. Int J Gynecol Cancer. 2011; 21:92-99.

29. Uegaki K, Shimada M, Sato S, Deura I, Naniwa J, Oishi T, Itamochi H, Harada T, Kigawa J. Outcome of stage IB2-IIB patients with bulky uterine cervical cancer who underwent neoadjuvant chemotherapy followed by radical hysterectomy. Int J Clin Oncol. 2014; 19:348-353.

30. Bradbury M, Founta C, Taylor W, Kucukmetin A, Naik R, Ang C. Pathological Risk Factors and Outcomes in Women With Stage IB2 Cervical Cancer Treated With Primary Radical Surgery Versus Chemoradiotherapy. Int J Gynecol Cancer. 2015; 25:1476-1483.
31. Liu Y, Zhao LJ, Li MZ, Li MX, Wang JL, Wei LH. The Number of Positive Pelvic Lymph Nodes and Multiple Groups of Pelvic Lymph Node Metastasis Influence Prognosis in Stage IA-IIB Cervical Squamous Cell Carcinoma. Chin Med J (Engl). 2015; 128:2084-2089.

32. Wang J, Wang T, Yang YY, Chai YL, Shi F, Liu ZI. Patient age, tumor appearance and tumor size are risk factors for early recurrence of cervical cancer. Mol Clin Oncol. 2015; 3:363-366.

33. Park JJ, Kim CK, Park SY, Park BK. Parametrial invasion in cervical cancer: fused T2-weighted imaging and highb-value diffusion-weighted imaging with background body signal suppression at 3 T. Radiology. 2015; 274:734-741.

34. Kinkel K. Pitfalls in staging uterine neoplasm with imaging: a review. Abdom Imaging. 2006; 31:164-173.

35. Yamanoi K, Matsumura N, Kido A, Baba T, Hamanishi J, Yamaguchi K, Yoshioka Y, Abou Taleb H, Togashi K, Konishi I. A novel diagnostic criterion for lymph node metastasis in cervical cancer using multi-detector computed tomography. Gynecol Oncol. 2013; 131:701-707.

36. Downey K, Shepherd JH, Attygalle AD, Hazell S, Morgan VA, Giles SL, Ind TE, Desouza NM. Preoperative imaging in patients undergoing trachelectomy for cervical cancer: validation of a combined T2- and diffusion-weighted endovaginal MRI technique at 3.0 T. Gynecol Oncol. 2014; 133:326-332. 